\title{
Genetic heterogeneity of benign thyroid lesions
}

Static and flow cytometry, karyotyping and in situ hybridization analysis

\author{
O. Ferrer-Roca ${ }^{\text {a }}$, J.A. Pérez-Gómez ${ }^{\mathrm{a}}$, J.C. Cigudosa ${ }^{\mathrm{b}}$, E. Gómez ${ }^{\mathrm{a}}$ and M. Estévez ${ }^{\mathrm{a}}$ \\ ${ }^{\mathrm{a}}$ Department of Pathology and ${ }^{\mathrm{b}}$ Department of Cytogenetics, Faculty of Medicine, \\ University of La Laguna, La Cuesta, 38071, La Laguna, Tenerife, Canary Islands, Spain \\ Tel.: 3422 642015; Fax: 3422 641855; E-mail: catai@redkbs.com
}

Received 2 June 1997

Revised 3 November 1997

Accepted 2 January 1998

\begin{abstract}
The present series includes 75 thyroid lesions (38 goiters, 30 adenomas, 3 follicullo-papillary encapsulated carcinomas and 4 normal thyroid) that were studied by static and flow cytometry. Four cases were also analyzed by in situ hybridization (centromeric probes for chromosomes 1 and 17) and 10 cases by G-banding cytogenetics.

Results demonstrate a polymorphysm and genetic instability in the thyroid tissue that may be related to the spontaneous polyploidization of their cells. The most consistent finding in cytometry was the presence of two clones associated with clinical or histological hyperactivity ( $46 \%$ versus $23 \%$ in non-functioning cases; $\chi^{2}$ distribution with a $p<0.05$ ).

Chromosomal anomalies were detected in two out of 10 cases: $46, \mathrm{XX}, \mathrm{t}(5,19)$ in $87 \%$ of cells of a diffuse hyperplastic goiter and $49, \mathrm{XX},+7,+17,+22$ in $19 \%$ of cells of thyroiditis case. Finally, the in situ hybridization technique showed hidden trisomies of clonal origin in all of the cases studied.

Evaluation of clonal trisomies by the in situ hybridization technique using the confidence interval of a binomial distribution is discussed.
\end{abstract}

Keywords: Thyroid, benign tumours, karyotyping, in situ hybridization, trisomies, clonal detection, chromosome 1, chromosome $17, \mathrm{t}(5,19)$

\section{Introduction}

The tendency of the endocrine normal tissue and tumours to have polyploid cells that produce a typical histological pattern with evident karyometric differences is well known $[5,11,30,35]$.

Those tetraploid stem-line karyotypes originating from polyploidization may produce a diploid or near-diploid stemline by chromosome segregation [10]; this fact was reproduced by computer modelling by Shackney et al. [28], in which duplication of chromosome set in the first phase is followed by subsequent losses.

This phenomenon is easy to study in thyroid, the reasons being: it is accessible, easy to grow in vitro and is subject to frequent hyperplasias, benign, and less frequently malignant tumours. Nervertheless, if the previous model is admissible, chromosomic segregation can be random, thus leading to scientific papers with a variety of results that are difficult to reproduce as well as evaluate. Furthermore, the 
sensitivity of thyroid to Iodine and its effect on the cell cycle, complicate the response of the thyroid tissue, since inorganic Iodide arrests the cells in G0G1 phase, while organified Iodide arrests the cells in G2M [31]; this, of course, may affect the spontaneous polyploidization and chromosomal segregation of the thyroid tissue. Finally, previous and recent studies demonstrate that most of the thyroid lesions are polyclonal, although in some cases a monoclonal origin can be detected [16,23].

Since 1970, we have been studying different cytogenetic and clonal aspects of benign thyroid lesions. According to our previous studies, that include the reorganization of the karyotypes in rats published by Al-Saadi et al. [1], Axelrad et al. [2] and our own results in humans (tissue culture arrested with colchicine during $24 \mathrm{~h}$ ), the benign thyroid lesions accumulate polysomies during their evolution from hyperplasia to goiter and adenomas. In our results it mainly affected $\mathrm{E}$ and $\mathrm{A}$ or $\mathrm{B}$ groups. The number of cells with $\mathrm{E}$ trisomies increases depending on the histological subtypes (13\% in thyroiditis, $17 \%$ in diffuse goiters, $19 \%$ in nodular goiters and $26 \%$ in adenomas). A or B trisomies were detected in nodular lesions (11\% of cells in nodular goitres, $23 \%$ in adenomas) [12-14]. The results of other authors agreed to classify the benign thyroid lesions in two groups: one characterized by a translocation at band $19 \mathrm{q} 13$ and the other characterized by hyperdiploid karyotypes with a basic trisomy in chromosome number 7 plus others affecting E, A or B groups [3,4].

Massive cell studies (flow cytometry and in situ hybridization) may or may not shed some light on the clonal evolution of thyroid lesions. Analysis of the literature in this field demonstrates heterogeneous results that detect a high degree of aneuploidies in benign and hyperplastic lesions [14,22,24, $26,27]$ with values of a range of $10 \%$ aneuploidies in goiters, $25 \%$ in adenomas, $60 \%$ in carcinomas, given to ploidy analysis an irrelevant prognostic value for thyroid carcinomas [6,22,25]. According to our knowledge, no papers on in situ hybridization have been published.

The present paper tries to evaluate the added value of interactive and flow DNA analysis, together with karyotyping and in situ hybridization in 75 thyroid lesions, most of them benign except three cases of encapsulated folliculo-papillary carcinomas.

\section{Material and methods}

We studied 75 thyroid lesions (prospective and retrospective analysis): 37 Goiters (5 Diffuse Hyperplastic (DHG), 30 Nodular Colloid (NCG), 2 Nodular Adenomatous (NAG)) as well as 27 adenomas (18 Simple Adenomas (SA), 3 Hürthler Adenomas (AH)), also 4 Normal Thyroids (TN) and 3 Folliculo-Papilar Carcinomas (CFP), the rest were mixed lesions such as: a NCG with a sclerosing follicular carcinoma, a SA in a thyroiditis, a SA plus a Hüthler adenoma and a AA coincidental with a follicular carcinoma.

The histological classification criterion is of relevance to evaluate the results. DHG were diffuse goiters with (Graves' disease) or without lymphoid infiltrates; NAG were nodular goiters with solid and cellular nodular lesions similar to fetal or embryonal adenomas but without the capsule. Adenomas include only solitary encapsulated lesions: AA were those adenomas having atypical cells and mitosis. AAI have, together with the previously stated situation, an invasion of the capsule, and CFP where cases of invasive follicular lesions with clear nuclei [18] and papillae in some areas. Finally, all lesions were classified as functioning or hyperplastic (F) or non-functioning (NF); the former due to clinical manifestations as well as pathological findings such as tall thyreocytes with or without papillae formation and reabsorption vacuoles in their colloid. From the whole of the series, 43 were classified as NF and 26 as F. In the NCG series, 11 were F and 17 NF (those cases not classified were due to difficulties in the histological sections or impossibility to review the slides). 
All cases were studied by static cytometry of the cells obtained from paraffin blocks by the Hedley method [19]; they were stained with progressive hematoxylin [17] and Feulgen; ploidy evaluated with internal controls, was obtained from the analysis of 100 to 300 cells. Flow cytometry was obtained from fresh tissue in 59 cases (see technique in [14]) and processed by a Coulter EPICS and/or by a Beckton-Dickinson FACScan.

In 10 cases (2 DHG, 4 NCG, 3 SA and $1 \mathrm{SA}+$ thyroiditis) fresh tissue was trypsinized and cultured in a RPMI-1640 media supplement with L-glutamine, $10 \%$ fetal calf serum and antibiotics. Karyotypes were prepared after $3 \mathrm{~h}$ of colchicine arrest $(0.02 \mu \mathrm{g} / \mathrm{ml})$ from a culture stimulated overnight with $30 \%$ fetal calf serum. Cells were processed by the standard trypsin-Giemsa technique for G-banding and a number of 20 to 45 mitoses were studied in each case.

Four cases (1 SA, 2 AAI, 1 NCG) were submitted to in situ hybridization with three biotinylated alfa satellite centromeric probes from ATCC (1, 17 and Y as a negative control); Walt's technique [34] was applied and visualized with hematoxylin in a 5 micron thickness histological section stained with peroxidase reaction and Diaminobenzidine (DAB). Evaluation of the results was done counting 200 nuclei per case; the criterion of Taguchi et al. [32] was used for one signal counting, due to non-resolved signals of the chromatids or cutting effect; the criterion of Jenderny et al. [20] was used for clonality when abnormalities were present in more then $3.6 \%$ of the nuclei. The impossibility to find normal tissue to compare, led us to evaluate the threshold by statistical means; threshold was the level by which the subtraction of the confidence interval (see statistics below) to the signal counting, gives $3.6 \%$ clonality values.

Statistical analysis includes $t$-test for continuous values, $\chi^{2}$ for the distribution of cases (except 0 and $100 \%$ distributions) and a Pearson linear correlation coefficient $(r)$. Significances were considered with $p<0.05$ in one tail test. Thresholding for abnormal signal counting was obtained from the confidence interval (standard deviation) of the binomial distribution (signal, non-signal) $\mathrm{SD}=Z \sqrt{(p q / n)}$, $Z$ being equal to 2.326 for an alfa of $0.01, n=$ number of counted nuclei, $p=$ probability of the signal and $q=1-p$. The interval chosen was for one tail test at 0.01 significance, because we wanted to reach the lower level of confidence on the abnormal signal countings. This will be referred to in the results as percentage minus the confidence interval for abnormal signals ( 3 and 4 ) and plus the confidence interval for normal diploid cells.

\section{Results}

The analysis of a double clonality in flow and static cytometry, as well as the presence of polyploid (4c) and aneuploid clones can be studied in Table 1.

Notice that the total number of cases in each column vary, because cases with flow and static cytometry are not coincidental and some static cytometry cases do not show internal controls. Figures 1 and 2 are examples of two clones in flow and static cytometry.

There was no difference between the frequency of two clones in goiters and adenomas $\left(\chi^{2}=0.84\right)$. Among the goiter group there is a significant difference between the $80 \%$ cases with two clones in diffuse (DHG) versus $27 \%$ in nodular goiters (NCG), $\chi^{2}$ of 9.14 and $p<0.0025$. Presence of two clones were also high in functioning versus non-functioning NCG with a $\chi^{2}$ of 4.26 and a $p<0.05$. This significance is mantained considering functionality versus non-functionality of the whole series ( $\chi^{2}$ of 3.91 and $p<0.05$ ). The effect of functionality is maximum if we compare DHG versus non-functioning NCG with a $\chi^{2}$ of 12.09 and $p<0.0001$. 
Table 1

Presence of biclonality, aneuploidy and polyploidy of the whole series

\begin{tabular}{lccccc}
\hline Histology & 2 clone FC & 2 clone SC & 2 clone total & Tetraploid c. & Aneuploid c. \\
\hline Goitres all & $8 / 30$ & $10 / 37$ & $12 / 37(32.4 \%)$ & $4 / 30(13 \%)$ & $6 / 30(20 \%)$ \\
DGH & $2 / 5$ & $4 / 5$ & $4 / 5(80 \%)^{* *}$ & $1 / 5(20 \%)$ & $2 / 5(40 \%)$ \\
NCG & $6 / 23$ & $6 / 30$ & $8 / 30(27 \%)$ & $3 / 23(13 \%)$ & $3 / 23(13 \%)$ \\
NAG & $0 / 2$ & $0 / 2$ & $0 / 2(0 \%)$ & $0 / 2(0 \%)$ & $1(\mathrm{SC}) / 2(50 \%)$ \\
NCG non-func. & $0 / 11$ & $1 / 17$ & $1 / 17(6 \%)^{*}$ & $0 / 11(0 \%)$ & $0 / 11(0 \%)$ \\
NCG functioning & $6 / 10$ & $5 / 11$ & $7 / 11(63.6 \%)$ & $3 / 10(30 \%)$ & $3 / 10(30 \%)$ \\
Adenoma all & $7 / 20$ & $13 / 28$ & $13 / 28(46 \%)$ & $3 / 20(15 \%)$ & $4 / 20(20 \%)$ \\
SA & $1 / 11$ & $4 / 19$ & $4 / 19(21 \%)$ & $0 / 11(0 \%)$ & $1 / 11(9 \%)$ \\
AA & $2 / 2$ & $1 / 2$ & $2 / 2(100 \%)$ & $0 / 2(0 \%)$ & $2 / 2(100 \%)$ \\
AH & $2 / 3$ & $2 / 3$ & $3 / 3(100 \%)$ & $2 / 3(66 \%)$ & $1 / 3(33 \%)^{\mathrm{a}}$ \\
AAI & $2 / 4$ & $1 / 4$ & $2 / 4(50 \%)$ & $1 / 4(25 \%)$ & $1 / 4(24 \%)$ \\
CFP & $0 / 3$ & $0 / 3$ & $0 / 3$ & $0 / 3$ & $0 / 3$ \\
All non-func. & $6 / 32$ & $7 / 43$ & $10 / 43(23 \%)^{*}$ & $3 / 32(9 \%)$ & $4 / 32(12.5 \%)$ \\
All functioning & $9 / 22$ & $10 / 26$ & $12 / 26(46 \%)$ & $3 / 22(14 \%)$ & $7 / 22(32 \%)$ \\
\hline
\end{tabular}

$\mathrm{SC}=$ static cytometry, $\mathrm{FC}=$ flow cytometry; ${ }^{*} p<0.05,{ }^{* *} p<0.005 ;{ }^{a}$ demonstrated in Fig. 1 . For abbreviations, see text $(\mathrm{DHG}=$ diffuse hyperplastic goiter; $\mathrm{NCG}=$ nodular colloid goiter; $\mathrm{NAG}=$ nodular adenomatous goiter; $\mathrm{SA}=$ simple adenoma; $\mathrm{AA}=$ atypical adenoma; $\mathrm{AH}=$ Hürthler adenoma; $\mathrm{AAI}=$ atypical adenoma with capsular invasion; $\mathrm{FPC}=$ folliculo-papillary carcinoma).

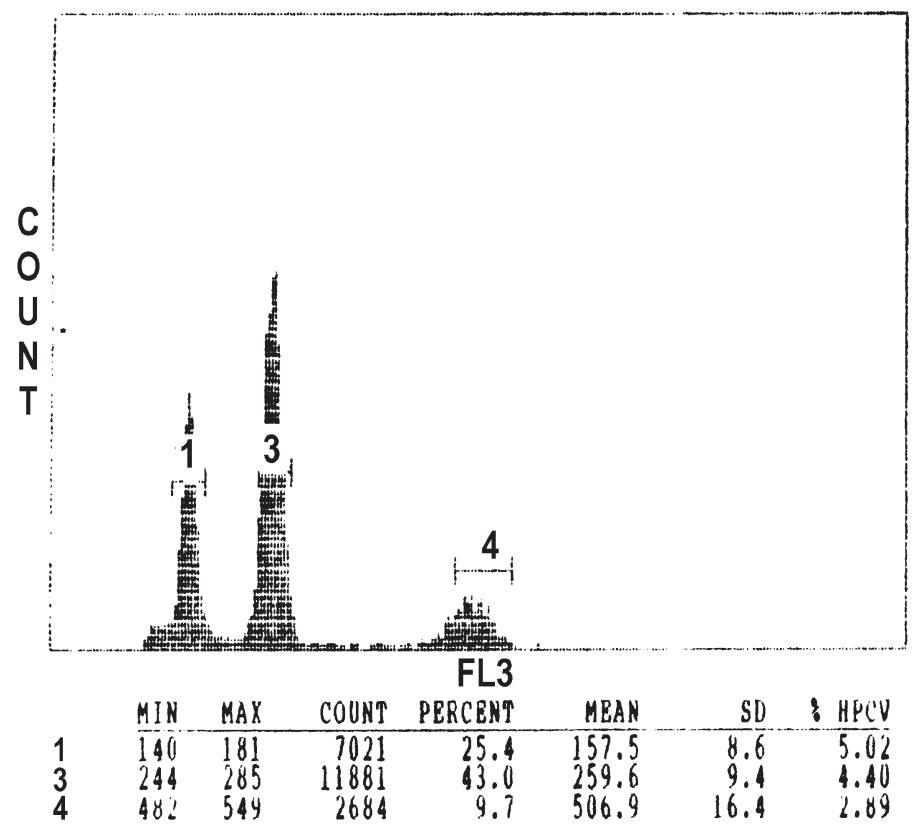

Fig. 1. Flow cytometry of case no. 41 of a biclonal Hürthler adenoma. A diploid (2c, peak 1) and aneuploid (3.4c, peak 3) clones; peak 4 is the G2M phase.

No significances were found in the subtypes of histological adenomas (AS versus AAI, $\chi^{2}$ of 1.27). On the remainder, the reduced number of cases and the extreme distribution does not allow $\chi^{2}$ analysis; nevertheless, those considered atypical adenomas (AA+AH) present a high rate of biclonality $(100 \%$; $5 / 5$ ), that decrease to $50 \%$ when the lesion becomes invasive (AAI).

The difference in distribution between polyploid and aneuploid cases was non-significant. 


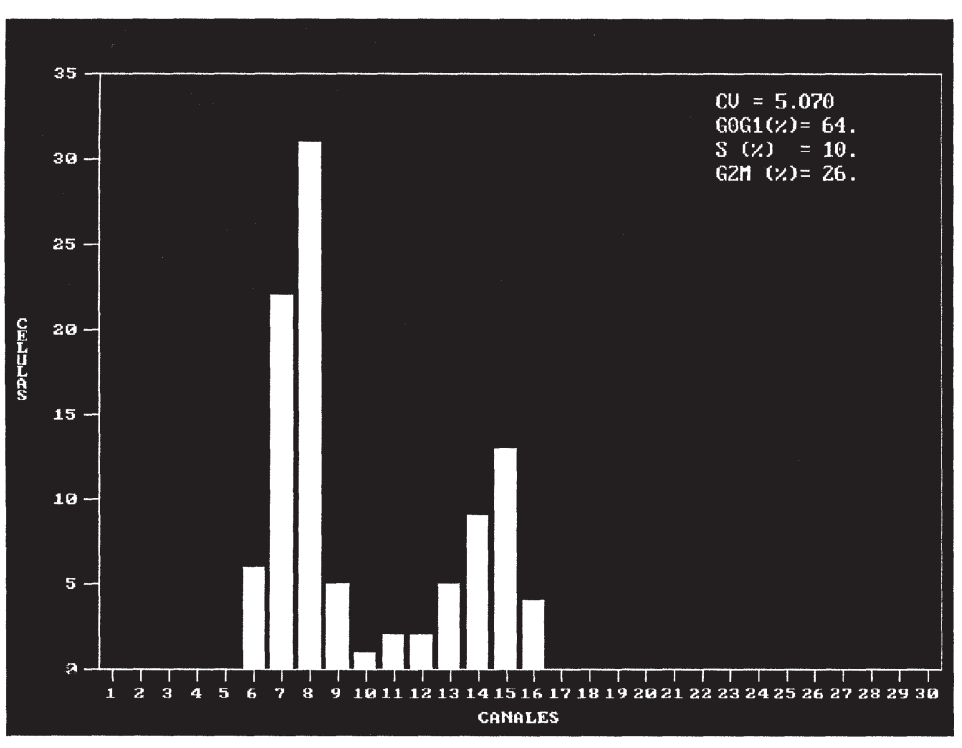

(a)

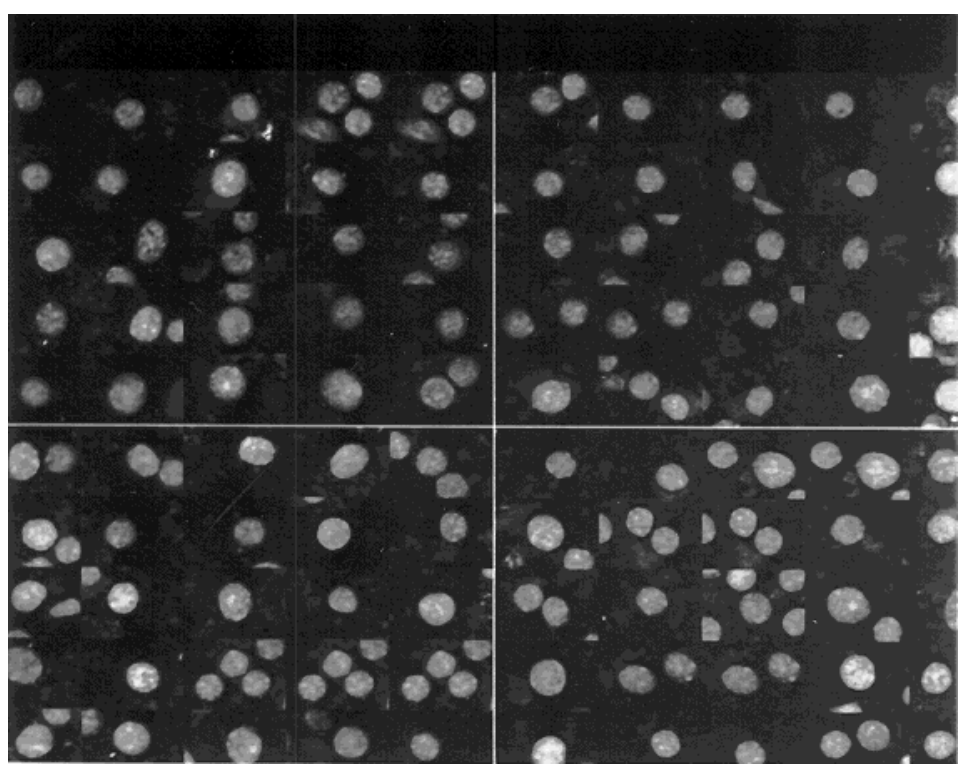

Fig. 2. Case no. 55 of a DHG with a balance translocation $t(5,19)$ [29] showing a second polyploid clone in the static cytometry (a), also clearly detected in morphometric (b). Optical density image obtained with the Texcan software (cf. O. Ferrer-Roca and J. Martin, Informatics in pathology. Software developed by the Texcan Group, in: Advances in Analytical Cellular Pathology, G. Burger, M. Oberholzer and G.P. Vooijs, eds, International Congress Series, Vol. 911, Excepta Medica, Amsterdam, 1990, pp. 41-42).

Cytogenetically two out of the 10 cases were abnormal. The case of SA with thyroiditis had a clone with a hyperdiploid karyotype $49, \mathrm{XX},+7,+17,+22$ ( 6 abnormal/26 normal diploid cells); see Fig. 3 . A case of a DGH, previously reported [7], showed a pseudodiploid clone with a reciprocal balance translocation, between chromosome 5 and 19, 46, XX, t(5,19) (q13,q13) (39 abnormal/6 normal diploid cells). Both cases had a normal flow cytometry. The static cytometry of the hyperdiploid case was normal and the $t(5,19)$ case showed a second polyploid clone; see Fig. 2. Using histology the 

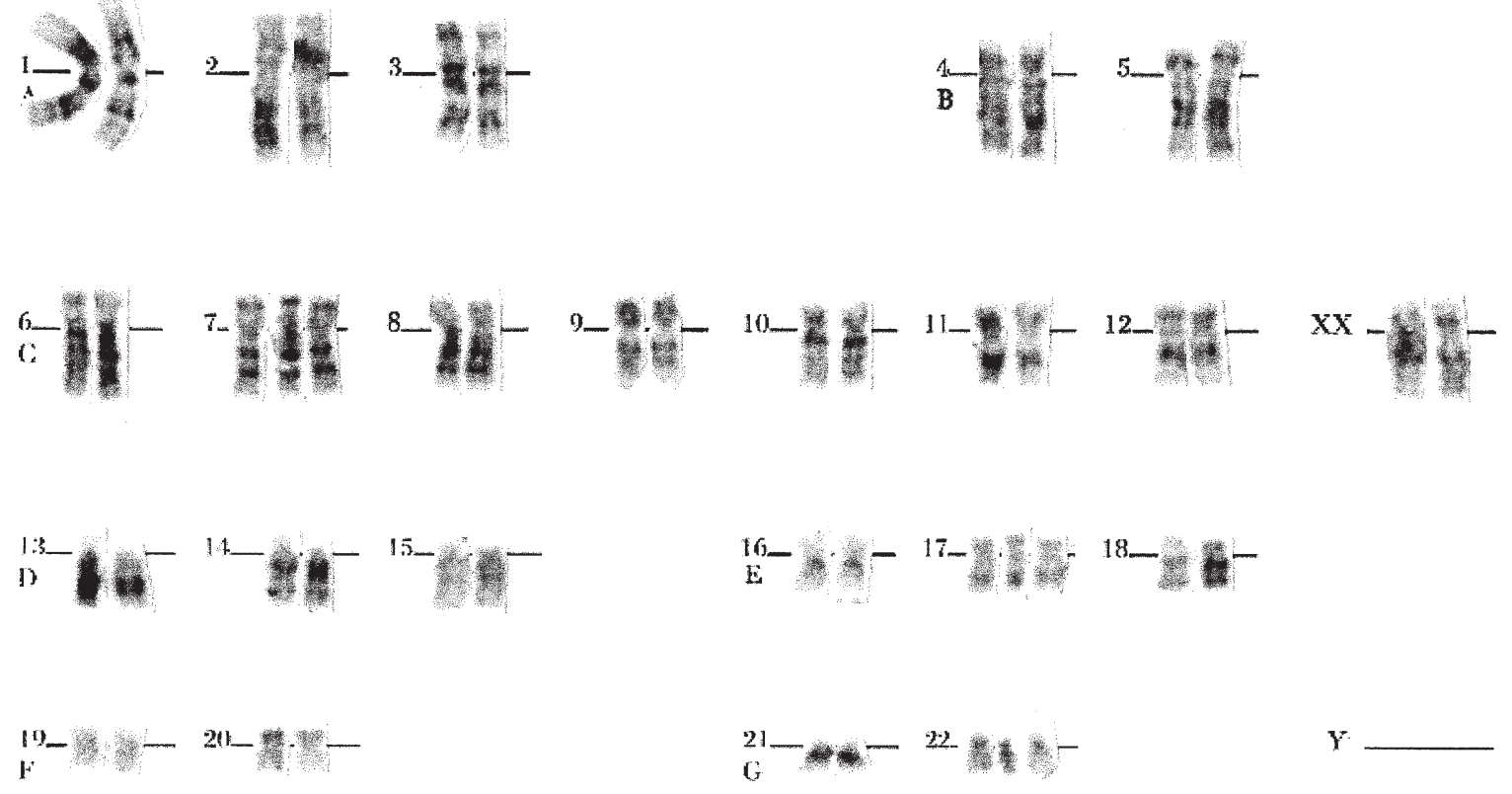

Fig. 3. A case of simple adenoma (SA) with thyroiditis, showing a hyperdiploid karyotype of $49, \mathrm{XX},+7,+17,+22$ in $25 \%$ of studied cells.

Table 2

Results on the in situ hybridization compared with flow cytometry

\begin{tabular}{lcccc}
\hline & Case no. 9 & Case no. 39 & Case no. 43 & Case no. 49 \\
\hline Ch1 2 signal & $90+7 \%$ & $83+8.7 \%$ & $76+9.9 \%$ & $92+6 \%$ \\
Ch1 3 signal & $8-6.3 \%$ & $\mathbf{1 1}-\mathbf{7 . 3 \%}$ & $\mathbf{1 6}-\mathbf{9 \%}$ & $6-5.5 \%$ \\
Ch1 4 signal & $2-4.5 \%$ & $6-5.5 \%$ & $8-6.3 \%$ & $4.6-2 \%$ \\
Ch17 2 signal & $74+10 \%$ & $91+6.6 \%$ & $55+11.6 \%$ & $82+9 \%$ \\
Ch17 3 signal & $\mathbf{1 5}-\mathbf{8 . 3 \%}$ & $2-4.6 \%$ & $\mathbf{1 5}-\mathbf{8 . 6 \%}$ & $\mathbf{1 4}-\mathbf{8 . 1 \%}$ \\
Ch17 4 signal & $\mathbf{1 1}-\mathbf{7 . 3 \%}$ & $7-5.9 \%$ & $\mathbf{3 0}-\mathbf{1 0 . 7 \%}$ & $4.5-4 \%$ \\
No. nuclei ch1/17 & $214 / 124$ & $128 / 141$ & $193 / 200$ & $208 / 102$ \\
Clones static c. & 1 & 1 & 1 & 1 \\
Clones flow c. & 1 & 1 & 1 & 1 \\
S-phase flow & $\mathbf{1 1 \%}$ & $8 \%$ & $\mathbf{1 2 \%}$ & $\mathbf{9 \%}$ \\
G2M-phase flow & $5.5 \%$ & $6 \%$ & $\mathbf{8 . 5 \%}$ & $2 \%$ \\
Diagnosis & $\mathrm{NCG}$ & $\mathrm{AAI}$ & $\mathrm{AAI}$ & $\mathrm{SA}$ \\
Functioning & $\mathrm{NF}$ & $\mathrm{NF}$ & $\mathrm{NF}$ & $\mathrm{F}$ \\
\hline
\end{tabular}

$\mathrm{NCG}=$ nodular colloid goiter; AAI = atypical adenome with capsular invasion; $\mathrm{SA}=$ simple adenoma.

Diffuse Hyperplastic Goitre (DHG) without focal lymphocytic infiltrates showed a hyperplastic thyroid tissue with papillae formation and prominent reabsortion vacuoles, having normal T3-T4 values. The thyroiditis case showed an extensive inflammation with oxyphylic transformation of thyreocytes and an encapsulated microfolicullar adenoma.

The in situ hybridization results, Figs 4 and 5, can be compared with the cytometric analysis in Table 2. In general, in situ hybridization detects more anomalies with chromosome 17, except case 39 in which we could not reach a substantial cell count. Applying the interval of confidence, trisomies of chromosome no. 1 were found in both atypical adenomas with capsular invasion, cases 39 and 43 


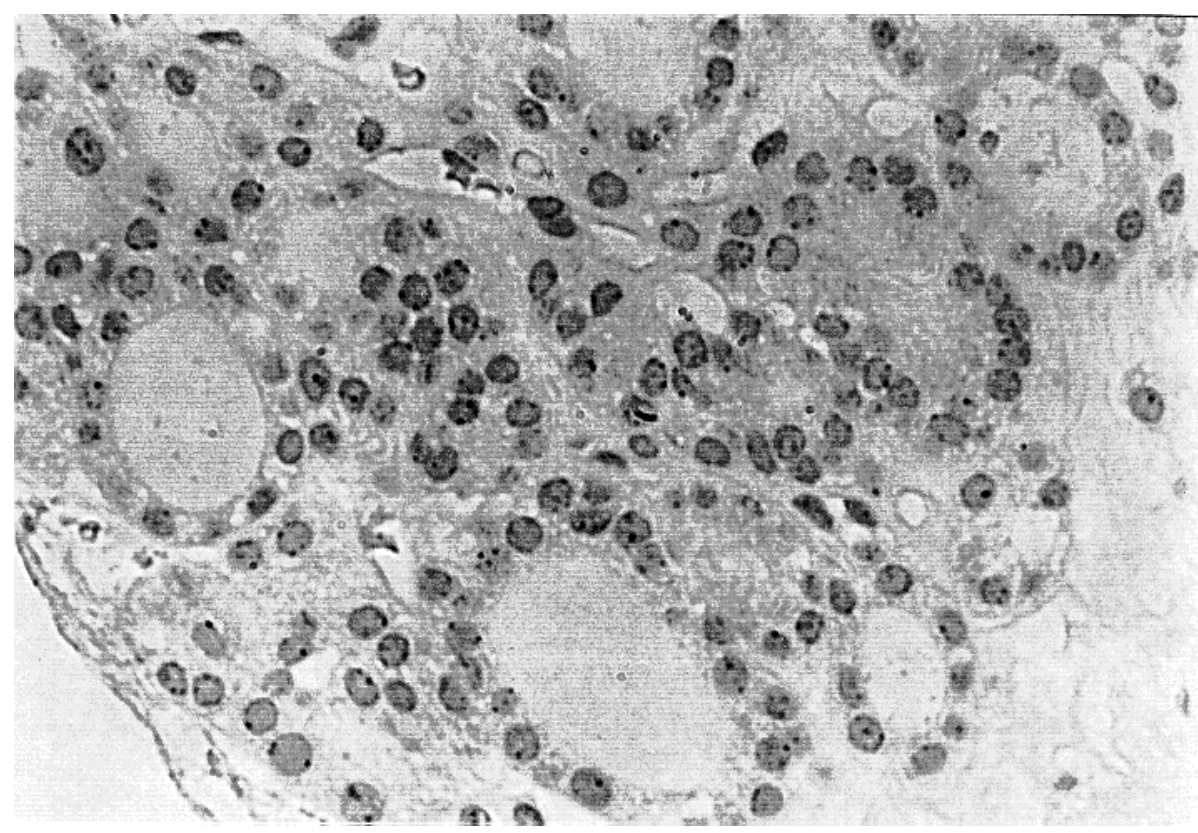

Fig. 4. In situ hybridization technique of a case of an atypical adenoma with capsular invasion (AAI, case no. 43) showing many trisomic cells with chromosome 17 probe.

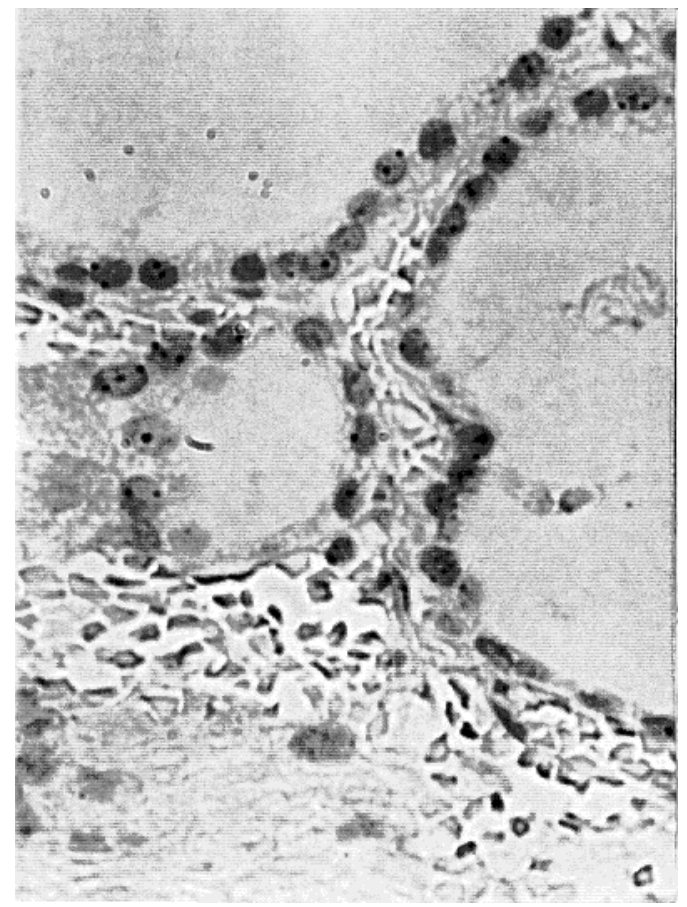

(a)

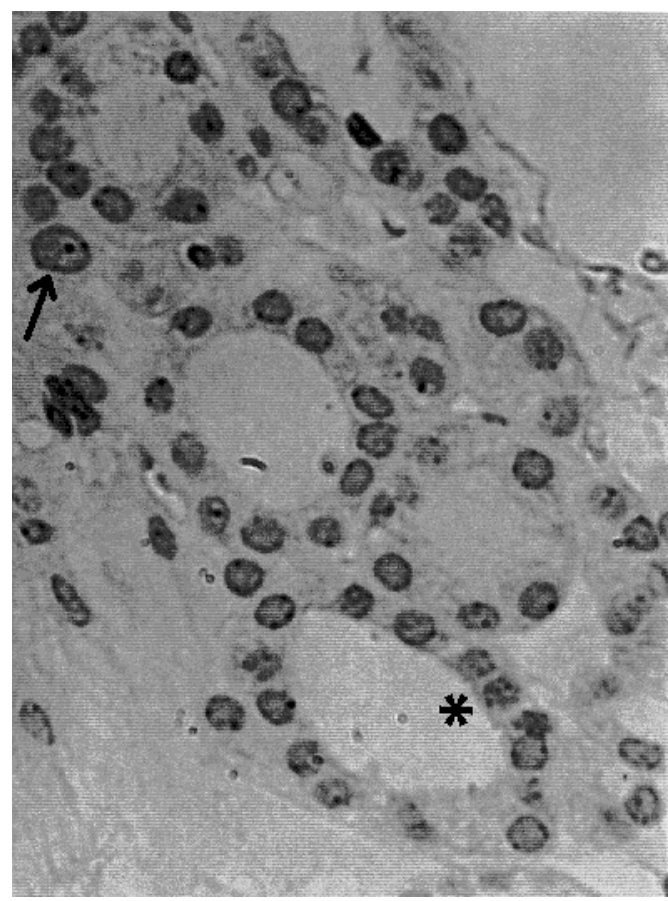

(b)

Fig. 5. (a) In situ hybridization of a nodular colloid goiter (NCG case no. 9) with chromosome 1 probe, showing two trisomic cells. (b) In situ hybridization of the AAI case no. 43 with chromosome 17 probe, that showed big, clear cells with two signals (arrow) and small cells with four signals $(*)$. 
(11-7.3\% and $16-9 \%$, respectively); and chromosome no. 17 trisomies in cases 49,43 and 9 associated with tetrasomies in the two latter cases.

Although the high number of tetrasomies in case $43(30-10.75 \%)$ were concordant with the high G2M on flow cytometry (8.5\%), the linear correlation between G2M flow phase and 4 signals or $\mathrm{S}$-phases with 3 signals cells were non-significant (ch1-G2M, $r=0.83$; ch1-S, $r=0.5 / \operatorname{ch} 17-\mathrm{G} 2 \mathrm{M}$, $r=0.85$; ch17-S, $r=0.78$ ) due to the fewer number of cases.

Comparison with normal thyroid tissue was not possible because trisomies were also detected in normal thyroid near adenomas (data not shown). It is also relevant to notice that the size of cells do not necessarily indicate polyploidization; on the contrary, many big, in general clear cells showed two signals while small dense cells present 4 signals (see Fig. $5(\mathrm{~b})$ ).

\section{Discussion}

The present DNA and chromosome study of the 75 thyroid lesions, most of them benign, demonstrates a genetic instability and polymorphism in the thyroid tissue linked to histological subtypes and functionality $(p<0.05)$.

Depending on the histological subtype, goitres showed a higher number of double clones on DHG $(p<0.0025$ and $p<0.0001$ versus all NCG and non-functioning NCG) which may indicate that the autoimmunity factor could also play a role. Based on this polymorphism, sampling as well as DNA karyotyping techniques could only be taken as complementary; this statement is clearly demonstrated in the DHG no. 55 with a balance translocation $\mathrm{t}(5,19)$, normal flow and biclonality on static cytometry (Fig. 2).

Functionality: globally speaking hyperfunctioning lesions have a double frequency of two clones $(p<0.05)$. Whether or not this is related with organic-inorganic Iodide equilibrium [31] is difficult to assess.

In the case of adenomas, the histological subtype could also be relevant. According to our results those considered as atypical (AA+AH) showed higher incidence of two clones that partially disappear in invasive cases, maybe as a clonal selection indicator. Unfortunately, the reduced number of cases and the extreme distribution of the present series does not allow statistical evaluation.

The number of polyploid and aneuploid clones is similar in the whole series, and despite the lack of statistical significance, aneuploidy is three times higher in functioning lesions.

The genetic instability of the thyroid tissue is also demonstrated in the two cases in which the clonal chromosomal abnormalities were not coincidental with flow or static cytometry. In one case, the thyroiditis component may play a role in their chromosomal abnormalities [15]. Both chromosomal anomalies belong to the groups outlined by Belge et al. [3,4]: the DGH with a translocation at band $19 \mathrm{q} 13$ in $87 \%$ of the cells and the SA plus thyroiditis with polysomies (19\% cells) including trisomies of chromosome no. 7 that is known to contain the erb-B1 oncogene [33]. These findings were in agreement with our previous results of $25 \%$ of cells with polysomies in adenoma cases [13].

Presence of trisomies have been reported in normal tissue, particularly trisomy of chromosome no. 7 $[8,9]$; therefore, the association of a specific polisomy in the pathogenesis of benign and malignant changes should be taken cautiously since it might only demonstrate a normal genetic heterogeneity.

Due to the high number of polysomies in the in situ hybridization techniques of the present series ( $100 \%$ cases with $14 \%$ of cells affected), if compared with karyotyping (10\% cases -1 case), we might conclude that, based on the genetic instability demonstrated by thyroid tissue, none of the results published can be discounted; they only show partial aspects of thyroid tissue behaviour. Also it could 
prove that the techniques themselves may play a role in selecting anomalies. For example: tissue culture under prolonged colchicine arrest may accumulate abnormal cells entering in mitosis; growing media, lacking inorganic Iodide may influence Iodide organification of thyreocytes and modify the G0-G1/G2M cell population balance with chromosomal segregation and detection of higher number of polysomies depending on cell differentiation and duration of the in vitro culture.

Under these circumstances the in situ hybridization technique could help to find the real incidence of polysomies and replications. From a methodological point of view, clonality should consider the interval of confidence of a binomial distribution; in the present results this technique showed the level of clonality to be at around $10 \%$ of the affected cells. This threshold also avoids the over-interpretation of the well documented non-specific hybridization [29]. A correct histological classification with regards to functionality and nodularity is strictly necessary for a coherent interpretation of DNA and chromosomal analysis of thyroid lesions. A clear example is shown in the already reported case with $t(5,19)$ of the present series whose published images [7] clearly showed tall cylindric hyperplastic thyreocytes with numerous reabsorption vacuoles and their macroscopic nodularity was not confirmed under histology, being reclassified in the present series as DHG.

Finally, the genetic instability demonstrated by the thyroid could probably be applied to other endocrine organs that spontaneously manifest a tendency towards polyploidization [21]. It also enforces the collateral conclusion of the inadequacy of thyroid and other hormone-dependent tissues as a control [20] for in the in situ hybridization techniques.

\section{Acknowledgements}

We would like to thank the collaboration of our secretary A. Tejera and technician J.P. Spencer, together with Dr. A. Cardona and Dr. Hassan for their cooperation in the tisssue culture and in situ hybridization techniques and Prof. Dr. H. Alvarez-Argüelles for the prospective cases in flow cytometry.

This work was carried out with the aid of a Grant given by the Regional Government of the Canary Islands no. 9/91.

\section{References}

[1] A.A. Al-Saadi and W.H. Beierwaltes, Chromosomal changes in rat in thyroid cells during iodine depletion and repletion, Cancer Res. 26 (1966), 676-688.

[2] A.A. Axelrad and C.P. Leblond, Induction of thyroid tumours in rats by low iodine diet, Cancer 8 (1955), 339-367.

[3] G. Belge, B. Thode, V. Rippe, S. Bartnizke and J. Bullerdiek, A characteristic sequence of trisomies starting with trisomy 7 in benign thyroid tumours, Hum. Genet. 94 (1994), 198-202.

[4] G. Belge, B. Thode, S. Bartnizke and J. Bullerdiek, Cytogenetic biclonality corresponding to multiphasic differentiation in an atypical adenoma, Cancer Genet. Cytogenet. 78 (1994), 102-104.

[5] L. Bondenson, A. Bondenson, O. Lindholm and S. Tibblin, Morphometric studies on nuclei in smears of fine needle aspirates from oxyphilic tumours of the thyroid, Acta Cytol. 27 (1983), 437-440.

[6] T. Bottger, D. Potratz, B. Schermus, J. Klupp, M. Stockle and T. Junginger, The value of quantitative DNA analysis in evaluating the prognosis of differentiated thyroid cancer, Chirurg. 65 (1994), 190-193.

[7] J.C. Cigudosa, A. Pedrosa Guerra, A. Otero Gómez, J.L. Carrasco Juan, J.A. Pérez Gómez, O. Ferrer-Roca and J.L. García Miranda, Translocation $(5,19)(\mathrm{q} 13, \mathrm{q} 13)$ in a multinodular thyroid goitre, Cancer Genet. Cytogenet. 82 (1995), 67-69.

[8] P. Elfving, J.C. Cigudosa, R. Lundgrem, U. Kristoffersson, N. Mandhal, S. Heim and F. Mitelman, Trisomy 7, trisomy 10, and loss of the Y chromosome in short-term cultures of normal kidney tissue, Cytogenet. Cell Genet. 53 (1990), 123-125. 
[9] P. Elfving, R. Lundgrem, J.C. Cigudosa, N. Mandhal, S. Heim and F. Mitelman, Trisomy 7 in non-neoplastic Kidney tissue cultured with and without EGF, Cancer Genet. Cytogenet. 64 (1992), 99-100.

[10] S.B. Ewers, E. Langstrom, B. Baldetorp and D. Killander, Flow-cytometric DNA analysis in primary breast carcinomas and clinicopathological correlations, Cytometry 5 (1984), 408-419.

[11] G.E. Feichter and K. Goerttle, Age-related nuclear size variability of thyreocytes in thyroid aspirates, Anal. Quant. Cytol. 5 (1983), 75-78.

[12] O. Ferrer-Roca and J. Egozcue, Estudio Etiopatogénico del cáncer y su importancia clinica V. Correlacion somaticoviral y teoria de la correlacion cariotipo-tumoural, Med. Clin. 65 (1975), 428-433.

[13] O. Ferrer-Roca, Clonal evolution of goiters, in: Tissue Culture in the Study of Tumors, Pacini, ed., Pisa, 1981, pp. $145-151$.

[14] O. Ferrer-Roca, E. Ballester-Guardia and J.A. Martin-Rodriguez, Morphometric, densitometric and flow cytometric criteria for the automated classification of thyroid lesions, Anal. Quant. Cytol. Histol. 22 (1990), 48-55.

[15] P.J. Fialkow, Autoimmunity and chromosomal aberrations, Am. J. Hum. Genet. 18 (1966), 93-108.

[16] P.J. Fialkow, The origin and development of human tumours studied with cell markers, New Engl. J. Med. 291 (1974), 26-35.

[17] G.W. Gill, J.K. Frost and K.A. Miller, A new formula for a half oxidased hematoxylin solution that neither overstains or requires differentiation, Acta Cytol. 18 (1974), 300-311.

[18] M.R. Hapke and L.P. Dehner, The optically clear nucleus. A reliable sign of papillary carcinoma of the thyroid?, Am. J. Surg. Pathol. 3 (1979), 31-38.

[19] D.W. Hedley, M.L. Friedlander, I.W. Taylor, C.A. Rugg and E.A. Musgrove, Methods for analysis of cellular DNA content of paraffin-embedded pathological material using flow cytometry, J. Histochem. Cytochem. 31 (1983), 13331335.

[20] J. Jenderny, E. Koster, A. Meyer, O. Borchers, W. Grote, D. Harms and U. Janing, Detection of chromosome aberrations in paraffin sections of seven gonadal yolk sac tumours of childhood, Hum. Genet. 96 (1995), 644-650.

[21] H. Joensuu and P.J. Klemi, DNA aneuploidy in adenomas of endocrine organs, Am. J. Pathol. 132 (1988), $145-151$.

[22] J.G. Jonasson and J. Hrafnkelsson, Nuclear DNA analysis and prognosis in carcinoma of the thyroid gland. A nation wide study in Iceland on carcinomas diagnosed 1995-1990, Virchows Arch. 425 (1994), 349-355.

[23] P. Kopp, E.T. Kimura, S. Aeschiman, M. Oestreicher, A. Tobler, M.F. Fey and H. Studer, Polyclonal and monoclonal thyroid nodules coexist within human multinodular goiters, J. Clin. Endocrin. Metab. 79 (1994), 134-139.

[24] G.L. Lukacs, G. Balazs, I. Zs-Nagy and M. Tivadar, Clinical meaning of DNA content in the long term behaviour of follicular thyroid tumours: a 12 years follow up, Eur. J. Surg. 160 (1994), 417-423.

[25] T. Oyhama, T. Ishida, K. Ishii, S. Sakurai, T. Joshita, A. Sakamoto and T. Nakajima, Encapsulated papillary carcinoma of the thyroid gland clinicopathological and cytofluorometric study in comparison with non-encapsulated papillary carcinoma, Acta Pathol. Jpn. 43 (1993), 516-521.

[26] T. Oyhama, A.L. Vickery, F.I. Preffer and R.B. Colvin, A comparative study of flow cytometry and histopathological findings in thyroid follicular carcinomas and adenomas, Hum. Pathol. 25 (1994), 271-275.

[27] I. Salmon, P. Gasperin, M. Remmelink, I. Rahier, P. Rocmans, J.L. Pastels, R. Heimann and R. Kiss, Ploidy level and proliferative activity measurements in a series of 407 thyroid tumours or other pathologic conditions, Hum. Pathol. 24 (1993), 912-920.

[28] S.E. Shackney, C.A. Smith, B.W. Miller, D.R. Burholt, K. Murthat, H.R. Giles, D.M. Ketteres and A.A. Pollice, Model for the genetic evolution of human solid tumours, Cancer Res. 49 (1989), 3344-3354.

[29] C.R. Schad and G.W. Dewald, Building a new clinical test for fluorescence in situ hybridization, Appl. Cytogenet. 21 (1995), 1-41.

[30] V. Smejkal, E. Smejkolova, M. Rosa, V. Zeman and K. Smetana, Cytologic changes simulating malignancy in Thyrotoxic Goiters treated with Carbimazole, Acta Cytol. 29 (1985), 173-178.

[31] P. Smerdely, V. Pitsiaves and S.C. Boyages, Evidence that inhibitory effects of iodide on thyroid cell proliferation are due to arrest of cell cycle at G0G1 and G2M phases, Endocrinology 133 (1993), 2881-2888.

[32] T. Taguchi, Z. Jian-Yuan, M. Feder, S. Litwin, A.J.P. Klein-Szanto and J.R. Testa, Detection of aneuploidy in interphase nuclei from non-small cell lung carcinomas by fluorescence in situ hybridization using chromosome-specific repetitive DNA probes, Cancer Genet. Cytogenet. 89 (1996), 120-125.

[33] E. Vand den Berg, J.W. Oosterhuis, B. De Jong, J. Buist, A.M. Vos, A. Dam and B. Vermeij, Cytogenetics of thyroid adenomas, Cancer Genet. Cytogenet. 44 (1990), 217-222.

[34] H. Walt, P. Emmerich, Th. Cremer, M.C. Hofmann and F. Babbwart, Supernumery chromosome 1 in interphase nuclei of atypical germ cells in paraffin-embedded human seminiferous tubules, Lab. Invest. 61 (1989), 527-531.

[35] J. Zajicek, Aspiration Biopsy Cytology: Part I. Cytology of Supradiaphragmatic Organs, G.L. Wied, ed., Monograph in Clinical Cytology, Vol. 4, S. Karger, Basel, 1974, p. 73. 


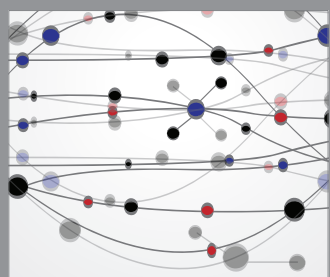

The Scientific World Journal
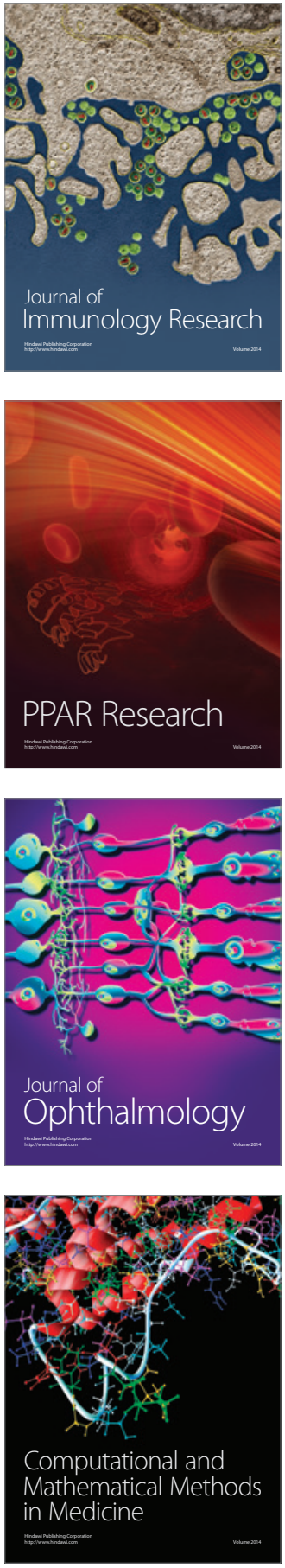

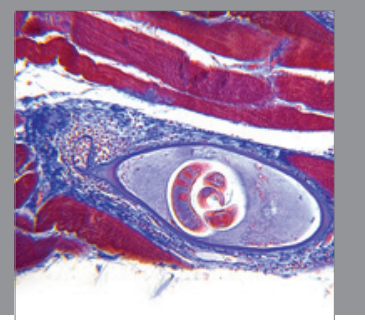

Gastroenterology

Research and Practice
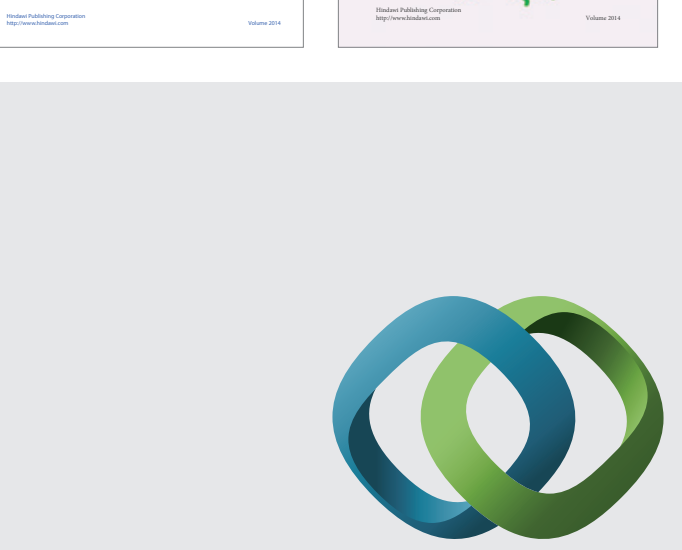

\section{Hindawi}

Submit your manuscripts at

http://www.hindawi.com
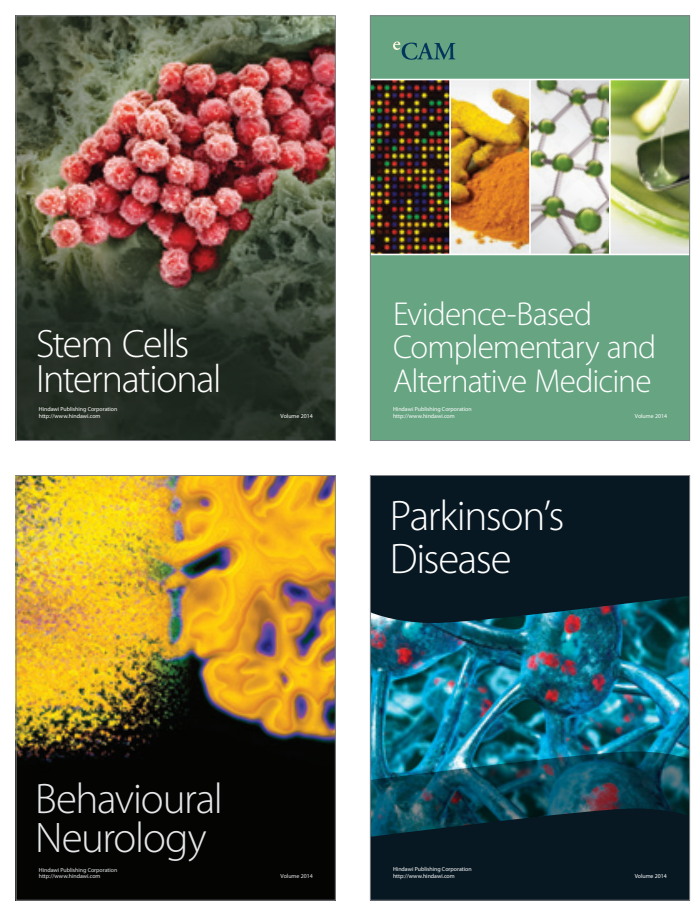

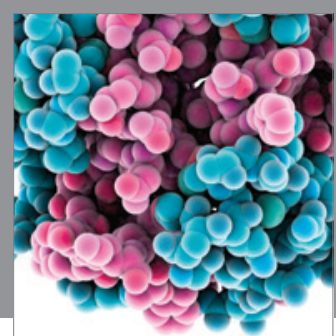

Journal of
Diabetes Research

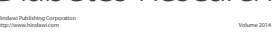

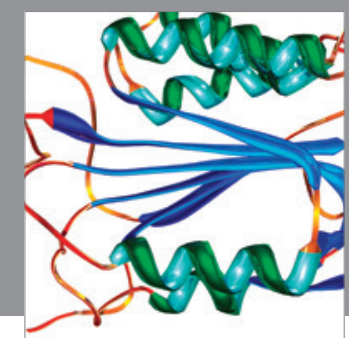

Disease Markers
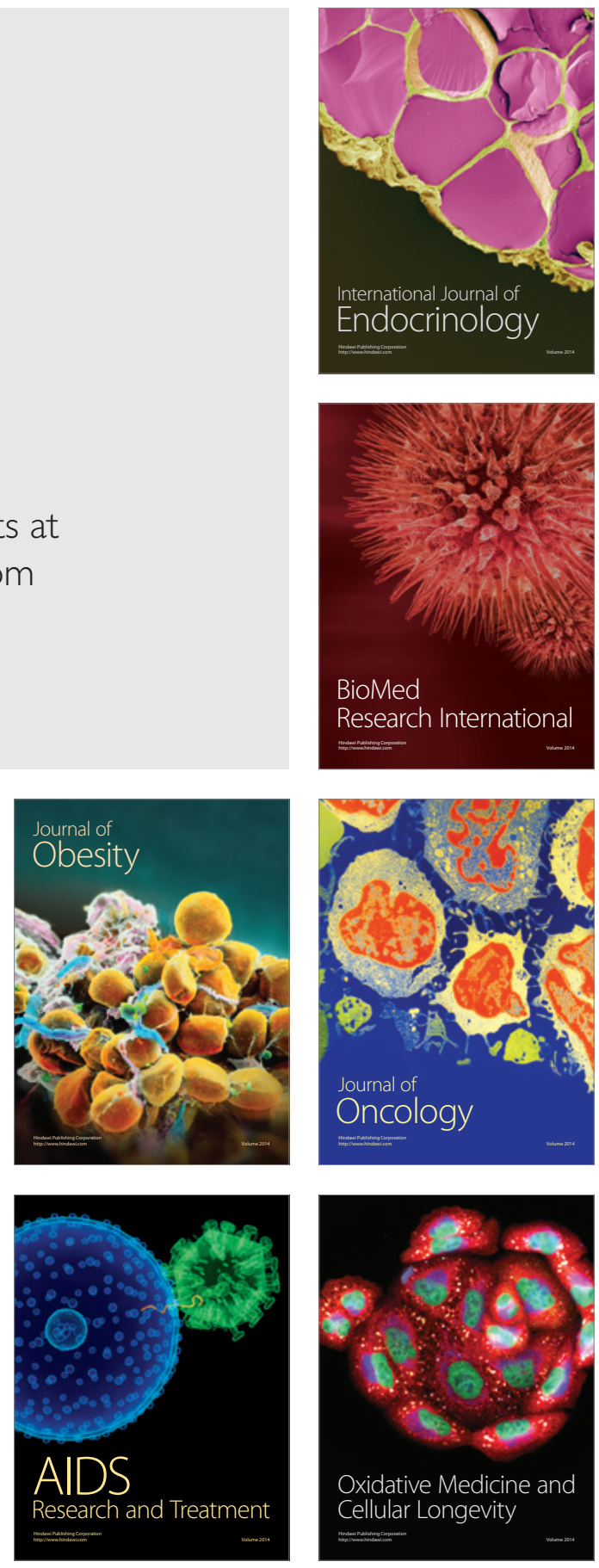